\title{
Une nouvelle ère pour la pédagogie de l'enseignement supérieur?
}

Christelle Lison

\section{(2) OpenEdition \\ 1 Journals}

\section{Electronic version}

URL: http://journals.openedition.org/ripes/2242

DOI: 10.4000/ripes.2242

ISSN: 2076-8427

\section{Publisher}

Association internationale de pédagogie universitaire

\section{Electronic reference}

Christelle Lison, «Une nouvelle ère pour la pédagogie de l'enseignement supérieur? », Revue internationale de pédagogie de l'enseignement supérieur [Online], 36(1) | 2020, Online since 11 April 2020, connection on 24 September 2020. URL : http://journals.openedition.org/ripes/2242 ; DOI : https:// doi.org/10.4000/ripes. 2242

This text was automatically generated on 24 September 2020 .

Article L.111-1 du Code de la propriété intellectuelle. 


\title{
Une nouvelle ère pour la pédagogie de l'enseignement supérieur?
}

\author{
Christelle Lison
}

1 L'année 2020 ne sera certainement pas une année comme les autres. Nous en sommes d'ores et déjà tous convaincus! Le coronavirus et ses impacts sur tout un chacun aura sans le moindre doute transformé notre vision du monde et de la formation. Il y a quelques semaines, nous avons pris la décision d'annuler l'édition 2020 du colloque de l'AIPU. Ce ne fût nullement une décision facile. J'en profite d'ailleurs pour remercier toutes les personnes qui ont travaillé fort au cours des deux dernières années pour la préparation de cet événement qui aurait été grandiose. Merci à Steve Vachon de l'Université Laval et à toutes les personnes, membres du comité d'organisation et du comité scientifique, qui se sont investies sans compter.

2 Par ailleurs, depuis quelques semaines, nous comprenons combien l'enseignement et l'apprentissage doivent prendre de multiples formes. Peut-on réellement imaginer que cette pandémie mondiale à laquelle nous faisons face n'aura aucune répercussion pour l'avenir des établissements d'enseignement supérieur? Peut-on réellement imaginer que cette situation ne se reproduira pas? Même si tout un chacun fait preuve de bonne volonté, il n'est néanmoins pas facile, pour les enseignants et les étudiants, de changer les choses en cours de semestre. C'est une occasion de développement professionnel sur laquelle il nous faudra réfléchir pour en tirer des leçons pédagogiques et didactiques. Au cours des dernières semaines, de nombreux établissements ont proposé de partager leurs réflexions et leurs outils pour favoriser le développement de stratégies pédagogiques adaptées. Ce premier numéro RIPES de l'année 2020, et les neuf articles qui le composent, peuvent eux aussi être sources d'inspiration pour les acteurs du monde de l'enseignement supérieur.

3 Le premier article est celui d'une équipe de chercheurs internationale (Québec et France). Louise Ménard et son équipe ont réalisé une recherche portant sur la formation des nouveaux professeurs, et plus spécifiquement sur les effets de celle-ci sur le niveau de centration sur l'apprentissage. Menée durant trois années, auprès de 34 
professeurs français et canadiens, cette recherche démontre la complexité du processus de développement pédagogique.

4 Le deuxième article, rédigé par Magali Hardouin de l'Université de Bretagne Occidentale, s'intitule Institutions postsecondaires françaises et pratiques d'internationalisation différenciées: analyse à partir du programme européen de doctorat international en réseau ERASMUS MUNDUS. En questionnant l'inégal accès des établissements d'enseignement supérieur au processus d'internationalisation en mettant à jour des pratiques, des logiques et des stratégies d'établissements variées, l'article montre qu'il existe des différences entre les établissements d'enseignement supérieur français dans leur capacité même à participer à un doctorat ERASMUS MUNDUS.

5 Le troisième article, proposé par Jean Vannereau (Université de Bordeaux) et Denis Lemaître (ENSTA Bretagne) vise à caractériser l'activité de problématisation dans l'apprentissage du management chez les étudiants en filière d'ingénieur en France, et ce, à travers l'étude de leurs productions. Il définit tout d'abord ce que l'on entend par situations managériales et problématisation. À l'issue de l'analyse, les auteurs mettent en évidence les différences dans la manière dont les étudiants cadrent les problèmes étudiés et proposent de relier le cadrage des problèmes à la posture intellectuelle et sociale adoptée par les étudiants.

6 Le quatrième article est celui de Julien Masson et de Laura Ratenet de l'Université Claude Bernard Lyon1. La recherche présentée dans cet article concerne le développement et la validation d'une échelle de mesure du sentiment d'efficacité personnelle des étudiants qui entrent à l'université. Testée auprès de près de 700 étudiants inscrits en première année dans 13 universités différentes, l'échelle permet de faire des liens avec les stratégies de coping mises en place par les étudiants.

7 Le cinquième article est celui d'Isabelle Cabot et Stéphanie Facchin, professeures dans des établissements supérieurs au Québec. Intitulée Identification des raisons de nonfréquentation des centres d'aide en français du postsecondaire au Québec, leur recherche a été réalisée à l'aide d'un questionnaire (985 répondants) et d'entrevues semi-dirigées (13 répondants). Les résultats montrent que les raisons d'ordre organisationnel, surtout relatives à la gestion du temps, sont les plus exprimées, mais des raisons d'ordre individuel, liées aux émotions ressenties, comme la honte ou la peur d'être jugé de façon négative, expliqueraient aussi une grande part la non-fréquentation des centres d'aide en français par les étudiants. À partir des résultats, les auteures en arrivent à proposer des pistes d'intervention mieux ajustées aux besoins des étudiants.

8 Le sixième article est celui d'Hélène Knoerr de l'Université d'Ottawa. L'étude qu'elle a réalisée porte sur un programme de parrainage offrant aux étudiants anglophones de première année inscrits dans une filière d'immersion universitaire en français la possibilité d'être associés à des étudiants francophones qui suivent le même cours de discipline en français. Le but de ce parrainage est triple: (1) améliorer leur réussite académique; (2) favoriser leur intégration sociale dans la communauté étudiante; (3) améliorer leurs compétences linguistiques en français. À l'issue de la recherche, les objectifs de l'activité semblent être atteints de manière satisfaisante.

9 Le septième article, intitulé Développer la recherche d'information et l'autorégulation collective en classe renversée: l'apport des tests d'autoévaluation, est proposé par Laetitia Thobois Jacob et Emmanuelle Chevry Pébayle, toutes deux de l'Université de Strasbourg. Dans le cadre d'une recherche exploratoire, les auteures ont mise en place 
une classe renversée dans un cours de Culture numérique auprès d'étudiants de première année. Elles ont ajouté au dispositif deux types de tests à visée formative (questions à choix multiples et questions à réponse libre). Les résultats montrent certains gains au niveau des apprentissages et le fait que les questions à réponse libre incitent les étudiants à mettre en place des stratégies d'autorégulation collective.

10 Le huitième article porte également sur un dispositif que l'on peut qualifier d'innovant, soit le dispositif de classe inversée PedagInnov mis en place afin de favoriser l'engagement des étudiants. Ayant réalisé cette recherche au sein de la Communauté d'universités et établissements de l'Université Paris-Est, Laetitia Gerard et Ariadna Ayala Rubio en arrivent à la conclusion que trois principales sources d'influence pédagogiques et didactiques influent sur l'engagement des étudiants: la posture d'accompagnateur, les ressources et les supports ainsi que l'évaluation.

11 Le neuvième article, proposé par Marie Machouart du Centre Hospitalier Régional Universitaire de Nancy, s'intitule Réveil des talents à l'université et place de l'enseignantcoach. À travers son texte, l'auteure nous amène à repenser nos méthodes pédagogiques et à réfléchir à l'évolution incontournable de notre rôle d'enseignant, notamment dans la perspective de devenir un coach, et ce, plus encore afin d'aider les étudiants qui vivent des situations difficiles. 